\title{
Metastatic Salivary Gland Carcinoma
}

National Cancer Institute

\section{Source}

National Cancer Institute. Metastatic Salivary Gland Carcinoma. NCI Thesaurus. Code C158463.

A salivary gland carcinoma that has metastasized to another anatomic site. 